\title{
POINT PROCESSES AND THE INFINITE SYMMETRIC GROUP
}

\section{Alexei Borodin and Grigori Olshanski}

\section{Introduction}

Our work originated from a problem of harmonic analysis on the infinite symmetric group $S(\infty)$.

The central theme of noncommutative harmonic analysis is decomposition of natural unitary representations $T$ into elementary ones (i.e., into irreducible or factor representations). When $T$ is endowed with a distinguished cyclic vector, its decomposition is governed by a measure, called the spectral or Plancherel measure. For instance, one of the achievements of the classical representation theory is the explicit calculation by Gindikin and Karpelevich of the spectral measure for the natural representation in the $L^{2}$ space on an arbitrary Riemannian symmetric space.

The group $S(\infty)$ consisting of finite permutations of the natural numbers is the simplest example of a big group whose elementary representations depend on infinitely many continuous parameters. In such a situation a spectral measure may be supported by an infinite-dimensional space - a phenomenon which did not arise in the classical representation theory.

In [KOV], a two-parameter family of generalized regular representations $T=$ $T_{z z^{\prime}}$ of $S(\infty)$ was constructed. They are realized in certain $L^{2}$ spaces on a compactification of $S(\infty)$. Their spectral measures, denoted as $P_{z z^{\prime}}$, live on an infinite-dimensional simplex $\Omega$ (a kind of dual object to $S(\infty)$ ). These are certain probability measures which are singular with respect to each other. The aim of the present work is to study the measures $P_{z z^{\prime}}$.

Our strategy is as follows. We embed the simplex $\Omega$ into a space of countable point configurations on the punctured real line $\mathbb{R}^{*}=\mathbb{R} \backslash\{0\}$; the points of a configuration accumulate near 0 . This enables us to interpret the measures $P_{z z^{\prime}}$ as certain point processes on $\mathbb{R}^{*}$. As the main tool for studying these processes we employ their correlation functions $\rho_{n}\left(x_{1}, \ldots, x_{n}\right), n=1,2, \ldots$

Received October 23, 1998.

Supported by the Russian Foundation for Basic Research under grant 98-01-00303 (G. O) and by the Russian Program for Support of Scientific Schools under grant 96-15-96060 (A. B. and G. O.) 
The correlation functions can be given by multidimensional integrals or expressed in terms of a multivariate hypergeometric series (the Lauricella function of type B). It turns out that their description is greatly simplified after a slight modification ('lifting') of the processes. Then the correlation functions take a determinantal form

$$
\rho_{n}\left(x_{1}, \ldots, x_{n}\right)=\operatorname{det}\left[K\left(x_{i}, x_{j}\right)\right]_{1 \leq i, j \leq n},
$$

with a certain kernel $K(x, y)$ on $\mathbb{R}^{*} \times \mathbb{R}^{*}$.

The explicit knowledge of the correlation functions enables us to derive a number of results about the properties of the measures $P_{z z^{\prime}}$. On the other hand, a rather special form (0.1) of the correlation functions makes it possible to understand the nature of the corresponding point processes and, consequently, of the initial measures. A variety of point processes with determinantal correlation functions arises in Random Matrix Theory (RMT, for short), and we were guided by this analogy.

We study in detail the kernel $K(x, y)$, which depends on the initial parameters $z, z^{\prime}$. It is expressed through the classical Whittaker functions. On the other hand, it can be expressed through another kernel $L$ which has a surprisingly simple form. ${ }^{1}$ Thus, it turns out that all the information about the initial measure $P_{z z^{\prime}}$ is encoded in this simple kernel $L$. It should be noted that the general structure of the kernel $K$ is similar to that of kernels arising in twomatrix models [EM].

We also show that the kernel $K$ admits various degenerations. They include the well-known in RMT sine and Bessel kernels as well as other Bessel-type kernels which, to our best knowledge, are new.

We believe that this work provides a new promising connection between RMT and Representation Theory.

A detailed exposition of the results is contained in five papers [Part I]-[Part V]. In the present paper we give a survey of the main results and indicate their location in [Part I]-[Part V].

\section{The Thoma simplex [Part I, $\S 1]$}

The starting point of our study was the work [KOV] on generalized regular representations of the infinite symmetric group. These representations depend on two parameters (further denoted by $z$ and $z^{\prime}$ ). The decomposition of the representations into irreducibles is governed by certain probability measures $P_{z z^{\prime}}$ living on the infinite-dimensional simplex

$$
\Omega=\left\{\alpha_{1} \geq \alpha_{2} \geq \ldots \geq 0 ; \beta_{1} \geq \beta_{2} \geq \ldots \geq 0 \mid \sum_{i=1}^{\infty}\left(\alpha_{i}+\beta_{i}\right) \leq 1\right\},
$$

\footnotetext{
${ }^{1}$ In terms of the corresponding integral operators, $K=L(1+L)^{-1}$.
} 
called the Thoma simplex [VK], [KV]. Note that $\Omega$ is compact in the topology of pointwise convergence.

Our aim is to understand these measures. Our results show that the measures $P_{z z^{\prime}}$ are close to stochastic point processes arising in scaling limits of random matrix ensembles.

\section{Probability measures $P_{z z^{\prime}}$ [Part I, $\left.\S \S 1,2\right]$}

The measures $P_{z z^{\prime}}$ are defined as follows. There exists a family $\left\{\widetilde{s}_{\lambda}\right\}$ of continuous functions on $\Omega$, indexed by the Young diagrams $\lambda$. Their linear span is dense in $C(\Omega)$. We know explicitly the integrals

$$
\varphi_{z z^{\prime}}(\lambda)=\int_{\Omega} \widetilde{s}_{\lambda}(\omega) P_{z z^{\prime}}(d \omega)
$$

which determine $P_{z z^{\prime}}$ uniquely. Essentially, this is the only information about $P_{z z^{\prime}}$ that we possess.

Now we shall describe the functions $\widetilde{s}_{\lambda}$ and write down the formula for $\varphi_{z z^{\prime}}$.

Let $\omega=(\alpha \mid \beta)$ range over $\Omega$. Set

$$
\widetilde{p}_{k}(\omega)= \begin{cases}1, & k=1, \\ \sum_{i=1}^{\infty} \alpha_{i}^{k}+(-1)^{k-1} \sum_{i=1}^{\infty} \beta_{i}^{k}, & k \geq 2,\end{cases}
$$

and for any partition $\rho=\left(\rho_{1}, \ldots, \rho_{l}\right)$,

$$
\widetilde{p}_{\rho}(\omega)=\widetilde{p}_{\rho_{1}}(\omega) \cdots \widetilde{p}_{\rho_{l}}(\omega)
$$

These are continuous functions on $\Omega$. The functions $\widetilde{s}_{\lambda}$ are related to $\widetilde{p}_{\rho}$ 's in exactly the same way as the Schur functions $s_{\lambda}$ are related to the power sums $p_{\rho}$, see $[\mathrm{M}, \S 1.7]$. Specifically,

$$
\widetilde{s}_{\lambda}=\sum_{\rho} \chi_{\rho}^{\lambda} z_{\rho}^{-1} \widetilde{p}_{\rho}
$$

where $\rho$ ranges over the partitions of $|\lambda|=\lambda_{1}+\lambda_{2}+\ldots ; \chi^{\lambda}$ is the irreducible character of the symmetric group of degree $|\lambda|, \chi_{\rho}^{\lambda}$ is its value on the conjugacy class indexed by $\rho$, and $z_{\rho}^{-1} \cdot|\lambda|$ ! is the cardinality of this conjugacy class.

Conversely,

$$
\widetilde{p}_{\rho}=\sum_{\lambda} \chi_{\rho}^{\lambda} \widetilde{s}_{\lambda}
$$

The functions $\widetilde{s}_{\lambda}$ are called extended Schur functions, see $[\mathrm{KV}]$. 
To define $\varphi_{z z^{\prime}}(\lambda)$ we need Frobenius notation for a Young diagram $\lambda$ :

$$
\lambda=\left(p_{1}, \ldots, p_{d} \mid q_{1}, \ldots, q_{d}\right) ;
$$

here $d$ is the number of diagonal boxes of $\lambda$, and

$$
p_{i}=\lambda_{i}-i, \quad q_{i}=\lambda_{i}^{\prime}-i,
$$

where $\lambda^{\prime}$ stands for the transposed diagram (see [M, §1.1]).

Set $n=|\lambda|, t=z z^{\prime}$. Then

$$
\begin{aligned}
\varphi_{z z^{\prime}}(\lambda)= & \\
\frac{t^{d}}{(t)_{n}} \prod_{i=1}^{d} \frac{(z+1)_{p_{i}}\left(z^{\prime}+1\right)_{p_{i}}(-z+1)_{q_{i}}\left(-z^{\prime}+1\right)_{q_{i}}}{p_{i} ! q_{i} !} & \\
& \times \frac{\prod_{i<j}\left[\left(p_{i}-p_{j}\right)\left(q_{i}-q_{j}\right)\right]}{\prod_{i, j}\left(p_{i}+q_{j}+1\right)},
\end{aligned}
$$

where $(a)_{m}=a(a+1) \cdots(a+m-1),(a)_{0}=1$.

We shall always assume that $z$ and $z^{\prime}$ satisfy one of the following conditions

(*) $\quad z^{\prime}=\bar{z}, z \in \mathbb{C} \backslash \mathbb{Z}$

(**) $z$ and $z^{\prime}$ are real and for a certain $m \in \mathbb{Z}, m<z, z^{\prime}<m+1$.

Under this assumption there exists a probability measure $P_{z z^{\prime}}$ such that $(2.1)$ holds.

Note that the measure $P_{z z^{\prime}}$ can be obtained as the limit, as $n \rightarrow \infty$, of certain statistics on partitions of $n$, see [KOO, proof of Theorem B], [Part II, Introduction].

\section{Stochastic point processes [Part I, $\S 4]$}

We shall interpret the measures $P_{z z^{\prime}}$ as stochastic point processes on $I=$ $[-1,1] \backslash\{0\}$. With each point $\omega \in \Omega$ we associate a point configuration in $I$,

$$
\omega=(\alpha \mid \beta) \mapsto\left(\alpha_{1}, \alpha_{2}, \ldots ;-\beta_{1},-\beta_{2}, \ldots\right),
$$

where we omit possible zeros in $\alpha$ and $\beta$. Note that any such configuration has no accumulation points in $I$, the points condensate near the origin which is not in $I$.

Thus, the measure $P_{z z^{\prime}}$ becomes a probability measure on the space of point configurations in $I$, i.e., a stochastic point process on $I$. We shall denote this process by $\mathcal{P}_{z z^{\prime}}$.

The $n$th correlation function $\rho_{n}\left(x_{1}, \ldots, x_{n}\right)$ of a point process is the density of the probability to find a point in each of the infinitesimal intervals $\left[x_{i}, x_{i}+d x_{i}\right]$ (see [DVJ], [Part I, §4] for details).

Our aim is to compute the correlation functions $\rho_{n}^{\left(z z^{\prime}\right)}$ of our processes $\mathcal{P}_{z z^{\prime}}$. 


\section{Moment problems [Part I, $\S 3]$, [Part II, Section 1.1]}

Our strategy to solve the problem is to find the probability measures $\sigma_{n}^{\left(z z^{\prime}\right)}$ on $[-1,1]^{n}, n=1,2, \ldots$, characterized by their moments

$$
\int_{[-1,1]^{n}} x_{1}^{l_{1}} \cdots x_{n}^{l_{n}} \sigma_{n}^{\left(z z^{\prime}\right)}(d x)=\int_{\Omega} \tilde{p}_{\left(l_{1}+1, \ldots, l_{n}+1\right)}(\omega) P_{z z^{\prime}}(d \omega) .
$$

Note that the RHS is explicitly known because of (2.1), (2.2):

$$
\int_{[-1,1]^{n}} x_{1}^{l_{1}} \cdots x_{n}^{l_{n}} \sigma_{n}^{\left(z z^{\prime}\right)}(d x)=\sum_{\lambda,|\lambda|=\sum_{i}\left(l_{i}+1\right)} \chi_{\left(l_{1}+1, \ldots, l_{n}+1\right)}^{\lambda} \varphi_{z z^{\prime}}(\lambda) .
$$

It turns out that outside the diagonals $x_{i}=x_{j}$ in $I^{n}$

$$
\rho_{n}^{\left(z z^{\prime}\right)}\left(x_{1}, \ldots, x_{n}\right)=\frac{1}{\left|x_{1} \cdots x_{n}\right|}\left\{\text { density of } \sigma_{n}^{\left(z z^{\prime}\right)}\left(d x_{1}, \ldots, d x_{n}\right)\right\}
$$

It is worth noting that the measure $\sigma_{n}^{\left(z z^{\prime}\right)}$ for $n \geq 2$ always has singular components living on the diagonals while (as is proved in [Part I, Proposition 4.2], [Part II, Theorem 2.5.1]) $\rho_{n}^{\left(z z^{\prime}\right)}$ has no such components ${ }^{2}$.

Thus, we obtain the correlation functions from a more sophisticated object. However, we can not formulate a moment problem for $\rho_{n}^{\left(z z^{\prime}\right)}$ directly because of the absolute value sign in (4.2).

\section{Integral representations for the correlation functions [Part I, $\S 5, \S 6]$, [Part II, Chapter 2]}

The moment problem (4.1) can be completely solved. In particular, for $n=1$ we obtained a two dimensional integral representation of $\sigma_{1}^{\left(z z^{\prime}\right)}$ (see [Part I, Theorem 5.8]). A careful examination shows that $\sigma_{1}^{\left(z z^{\prime}\right)}$ has no atom at zero. This fact has an important corollary.

Theorem I ([Part I, Theorem 6.1]). With probability 1,

$$
\sum_{i=1}^{\infty}\left(\alpha_{i}+\beta_{i}\right)=1
$$

For $n>1$ the solution of the moment problem (4.1) requires a lot of work ([Part II, Chapter 1]). The difficulties come from the fact that the RHS of (4.1)

\footnotetext{
${ }^{2}$ This just means that nonzero coordinates in $\alpha$ and $\beta$ are pairwise distinct with probability 1.
} 
involves the symmetric group characters for which there is no simple expression. We use Murnaghan-Nakayama rule $([\mathrm{M}, \S 1.7$, Ex. 5]) to handle the characters. In [Part II, Theorem 1.2.1'] we obtain a more economic form of this rule which enables us to solve the moment problem.

The final expression for $\rho_{n}^{\left(z z^{\prime}\right)}$ is a linear combination of multidimensional integrals of various orders up to $3 n$. The situation simplifies when all the variables $x_{1}, \ldots, x_{n}$ are of the same sign, say, positive.

Theorem II ([Part II, Theorem 2.2.1]). Let $x_{1}, \ldots, x_{n}>0$. Then

$$
\begin{aligned}
& \rho_{n}^{\left(z z^{\prime}\right)}\left(x_{1}, \ldots, x_{n}\right)=t^{n} \Gamma(t) \\
& \times \int_{\substack{a_{i}, b_{i}>0 \\
\sum_{i} x_{i}\left(a_{i}+b_{i}+1\right)<1}} \prod_{i=1}^{n} \frac{a_{i}^{-z}}{\Gamma(-z+1)} \frac{\left(a_{i}+1\right)^{z^{\prime}}}{\Gamma\left(z^{\prime}+1\right)} \frac{b_{i}^{-z^{\prime}}}{\Gamma\left(-z^{\prime}+1\right)} \frac{\left(b_{i}+1\right)^{z}}{\Gamma(z+1)} \\
& \quad \times \operatorname{det}\left(\frac{1}{a_{i}+b_{j}+1}\right) \frac{\left(1-\sum_{i} x_{i}\left(a_{i}+b_{i}+1\right)\right)^{t-n-1}}{\Gamma(t-n)} d a d b .
\end{aligned}
$$

The RHS of (5.1) is well defined for $z$ and $z^{\prime}$ such that

$$
-1<\Re z, \Re z^{\prime}<1 ; \quad t=z z^{\prime}>n .
$$

For other values of $z, z^{\prime}$ we use analytic continuation.

We tacitly assume that $\sum_{i} x_{i}<1$. Actually, the correlation measure $\rho_{n}^{\left(z z^{\prime}\right)}(x) d x$ always lives on the set $\sum_{i}\left|x_{i}\right| \leq 1$; additional considerations show that there are no singular components on the faces $\sum_{i}\left|x_{i}\right|=1$, see the beginning of the proof of Theorem 3.3.1 in [Part II].

The RHS of (5.1) can be expressed via the multivariate Lauricella hypergeo-

metric function of type B, see [Part II, Section 2.4]. In particular, $\rho_{1}^{\left(z z^{\prime}\right)}$ can be expressed in terms of the Appell's two-dimensional hypergeometric function $F_{3}$, see [Part II, Corollary 2.4.2]. Another expression of $\rho_{1}^{\left(z z^{\prime}\right)}$ through the Lauricella function in three variables is given in [Part I, Theorem 5.12].

\section{Lifting [Part II, Chapter 3]}

A surprising fact is that the correlation functions are greatly simplified after a 'lifting' of our processes $\mathcal{P}_{z z^{\prime}}$ to a slightly bigger space.

According to Theorem I, the measure $P_{z z^{\prime}}$ is concentrated on the face

$$
\Omega_{0}=\left\{\omega=(\alpha \mid \beta) \mid \sum_{i}\left(\alpha_{i}+\beta_{i}\right)=1\right\} \subset \Omega
$$


Let $\widetilde{\Omega}_{0}=\Omega_{0} \times \mathbb{R}_{+}$. We pass from the measure $P_{z z^{\prime}}$ on $\Omega_{0}$ to the measure

$$
\widetilde{P_{z z^{\prime}}}=P_{z z^{\prime}} \otimes\left\{\frac{s^{t-1}}{\Gamma(t)} e^{-s} d s\right\}
$$

on $\widetilde{\Omega}_{0}$. In other words, we tensor $P_{z z^{\prime}}$ by the gamma-distribution with parameter $t$.

We associate to a point $\widetilde{\omega}=(\omega, s) \in \widetilde{\Omega}_{0}$ a point configuration in $\mathbb{R}^{*}=\mathbb{R} \backslash\{0\}$ as follows

$$
((\alpha \mid \beta), s) \mapsto\left(\widetilde{\alpha}_{1}, \widetilde{\alpha}_{2}, \ldots ;-\widetilde{\beta}_{1},-\widetilde{\beta}_{2}, \ldots\right)=\left(s \alpha_{1}, s \alpha_{2}, \ldots ;-s \beta_{1},-s \beta_{2}, \ldots\right) .
$$

Thus, we get a probability measure on the space of point configurations in $\mathbb{R}^{*}$, i.e., a point process. We denote it by $\widetilde{\mathcal{P}_{z z^{\prime}}}$.

The process $\widetilde{\mathcal{P}_{z z^{\prime}}}$ is obtained from $\mathcal{P}_{z z^{\prime}}$ by multiplying the random configuration in $I$ by the scalar random factor $s$ with gamma-distribution.

The lifting is invertible via the map

$$
(\widetilde{\alpha} \mid \widetilde{\beta}) \mapsto(\alpha \mid \beta)=\left(\frac{\widetilde{\alpha}_{1}}{s}, \frac{\widetilde{\alpha}_{2}}{s}, \ldots ;-\frac{\widetilde{\beta}_{1}}{s},-\frac{\widetilde{\beta}_{2}}{s}, \ldots\right),
$$

where $s=\sum_{i}\left(\widetilde{\alpha}_{i}+\widetilde{\beta}_{i}\right)$.

Note that $s$ is finite almost surely with respect to $\widetilde{P_{z z^{\prime}}}$.

Let $\widetilde{\rho}_{n}^{\left(z z^{\prime}\right)}\left(x_{1}, \ldots, x_{n}\right)$ be the $n$th correlation function of $\widetilde{\mathcal{P}_{z z^{\prime}}}$.

The 'lifted' correlation functions are related to the initial ones by the following simple transformation

$$
\widetilde{\rho}_{n}^{\left(z z^{\prime}\right)}\left(x_{1}, \ldots, x_{n}\right)=\int_{0}^{\infty} \frac{s^{t-1} e^{-s}}{\Gamma(t)} \rho_{n}^{\left(z z^{\prime}\right)}\left(x_{1} s^{-1}, \ldots, x_{n} s^{-1}\right) \frac{d s}{s^{n}}
$$

see [Part II, Proposition 3.1.1]. This implies that the moments of the correlation measures $\rho_{n}^{\left(z z^{\prime}\right)}(x) d x$ and $\widetilde{\rho}_{n}^{\left(z z^{\prime}\right)}(x) d x$ are related in a very simple way

$$
\begin{aligned}
\int x_{1}^{l_{1}} \cdots x_{n}^{l_{n}} \widetilde{\rho}_{n}^{\left(z z^{\prime}\right)}\left(x_{1}, \ldots, x_{n}\right) d x_{1} \ldots d x_{n} & \\
& =(t)_{l_{1}+\ldots+l_{n}} \int x_{1}^{l_{1}} \cdots x_{n}^{l_{n}} \rho_{n}^{\left(z z^{\prime}\right)}\left(x_{1}, \ldots, x_{n}\right) d x_{1} \ldots d x_{n}
\end{aligned}
$$

(the moments are finite if $l_{1}, \ldots, l_{n} \geq 1$ ).

Note also that the transformation (6.1) is invertible, and there exists a complex inversion formula similar to that for the Laplace transform. 


\section{Determinantal formula. Matrix Whittaker kernel [Part IV, Sections 1,2]}

Now we shall show that the correlation functions $\widetilde{\rho}_{n}^{\left(z z^{\prime}\right)}$ have determinantal form with a kernel expressed through the Whittaker function $W_{\kappa, \mu}(x), x>0$.

This function can be characterized as the only solution of the Whittaker equation

$$
y^{\prime \prime}-\left(\frac{1}{4}-\frac{\kappa}{x}+\frac{\mu^{2}-\frac{1}{4}}{x^{2}}\right) y=0,
$$

such that $y \sim x^{\kappa} e^{-\frac{x}{2}}$ as $x \rightarrow+\infty$ (see [E1, Chapter 6]). Here $\kappa$ and $\mu$ are complex parameters. Note that

$$
W_{\kappa, \mu}=W_{\kappa,-\mu} .
$$

We shall employ the Whittaker function for real $\kappa$ and real or pure imaginary $\mu$; then $W_{\kappa, \mu}$ is real.

Set

$$
a=\frac{z+z^{\prime}}{2}, \quad \mu=\frac{z-z^{\prime}}{2}, \quad \sigma=\sqrt{\sin (\pi z) \sin \left(\pi z^{\prime}\right)}>0 .
$$

It is often convenient to consider $(a, \mu)$ as new parameters of our processes, instead of $\left(z, z^{\prime}\right)$. In terms of $(a, \mu)$ the basic assumptions (2.4) take the following form

- $a$ is always real

- either $\mu$ is pure imaginary

- or $\mu$ is real, $|\mu|<\frac{1}{2}$, and there exists $m \in \mathbb{Z}$ such that $m+|\mu|<a<$ $m+1-|\mu|$

- $a$ is not an integer when $\mu=0$.

Introduce the following functions on $\mathbb{R}_{+}$

$$
\begin{array}{ll}
A_{+}(x)=\frac{1}{\sqrt{x}} W_{a+\frac{1}{2}, \mu}(x), & A_{-}(x)=\frac{1}{\sqrt{x}} W_{-a+\frac{1}{2}, \mu}(x), \\
B_{+}(x)=\frac{1}{\sqrt{x}} W_{a-\frac{1}{2}, \mu}(x), & B_{-}(x)=\frac{1}{\sqrt{x}} W_{-a-\frac{1}{2}, \mu}(x) .
\end{array}
$$

Theorem III ([Part IV, Theorem 2.7]). The correlation functions of the lifted process $\widetilde{\mathcal{P}_{z z^{\prime}}}$ have the form

$$
\begin{gathered}
\widetilde{\rho}_{n}^{\left(z z^{\prime}\right)}\left(u_{1}, \ldots, u_{n}\right)=\operatorname{det}\left[K\left(u_{i}, u_{j}\right)\right]_{i, j=1}^{n}, \\
n=1,2, \ldots ; \quad u_{1}, \ldots, u_{n} \in \mathbb{R}^{*}
\end{gathered}
$$

where the kernel $K(u, v)$ is conveniently written in the block form

$$
K(u, v)= \begin{cases}K_{++}(u, v), & u, v>0 \\ K_{+-}(u,-v), & u>0, v<0 \\ K_{-+}(-u, v), & u<0, v>0 \\ K_{--}(-u,-v), & u, v<0\end{cases}
$$


with

$$
\begin{array}{r}
K_{++}(x, y)=\frac{1}{\Gamma(z) \Gamma\left(z^{\prime}\right)} \frac{A_{+}(x) B_{+}(y)-B_{+}(x) A_{+}(y)}{x-y}, \\
K_{+-}(x, y)=\frac{\sigma}{\pi} \frac{A_{+}(x) A_{-}(y)+t B_{+}(x) B_{-}(y)}{x+y}, \\
K_{-+}(x, y)=-\frac{\sigma}{\pi} \frac{A_{+}(y) A_{-}(x)+t B_{+}(y) B_{-}(x)}{x+y}, \\
K_{--}(x, y)=\frac{1}{\Gamma(-z) \Gamma\left(-z^{\prime}\right)} \frac{A_{-}(x) B_{-}(y)-B_{-}(x) A_{-}(y)}{x-y}
\end{array}
$$

(recall that $\left.t=z z^{\prime}\right)$.

This is one of our main results.

The matrix representation of the kernel

$$
K=\left[\begin{array}{ll}
K_{++} & K_{+-} \\
K_{-+} & K_{--}
\end{array}\right]
$$

correponds to the splitting $\mathbb{R}^{*}=\mathbb{R}_{+} \sqcup \mathbb{R}_{-}$of the phase space and subsequent identification of $\mathbb{R}_{-}$with the second copy of $\mathbb{R}_{+}$. We call (7.4) the matrix Whittaker kernel.

All the blocks of (7.4) are real valued kernels on $\mathbb{R}_{+}$. They possess the following symmetry

$$
K_{++}(x, y)=K_{++}(y, x), \quad K_{--}(x, y)=K_{--}(y, x), \quad K_{+-}(x, y)=-K_{-+}(y, x) .
$$

Note the minus sign in the last relation. It means that the kernel (7.4) is formally $J$-symmetric where $J$ is the operator id $\oplus($-id $)$ in $L^{2}\left(\mathbb{R}_{+}, d x\right) \oplus L^{2}\left(\mathbb{R}_{+}, d x\right)$.

Another symmetry property: the change of parameters $\left(z, z^{\prime}\right) \rightarrow\left(-z,-z^{\prime}\right)$ is equivalent to the transform of the kernel

$$
\left[\begin{array}{ll}
K_{++} & K_{+-} \\
K_{-+} & K_{--}
\end{array}\right] \longrightarrow\left[\begin{array}{cc}
K_{--} & -K_{-+} \\
-K_{+-} & K_{++}
\end{array}\right]
$$

Determinantal form for the correlation functions (like (7.2)) appears in different problems of random matrix theory and mathematical physics, see, e.g., [Dy, Me1, Ma1, Ma2, TW1-3, L, KBI]. In most situations the kernel $K$ is symmetric or Hermitian (see, however, $[\mathrm{B}]$ ). Appearance of $J$-symmetric kernels seems to be new.

\section{The $L$-kernel [Part $\mathrm{V}, \S 2]$}

Consider the operator $K$ in the Hilbert space

$$
L^{2}\left(\mathbb{R}^{*}, d u\right) \simeq L^{2}\left(\mathbb{R}_{+}, d x\right) \oplus L^{2}\left(\mathbb{R}_{+}, d x\right),
$$

given by the kernel (7.4). 
Theorem IV ([Part V, Theorem 2.4]). Assume $|a|<\frac{1}{2}$. Then

$$
K=\frac{L}{1+L}
$$

where $L$ is bounded and given by the kernel

$$
L(x, y)=\left[\begin{array}{cc}
0 & \frac{\sigma}{\pi}\left(\frac{x}{y}\right)^{a} \frac{\exp \left(-\frac{x+y}{2}\right)}{x+y} \\
-\frac{\sigma}{\pi}\left(\frac{y}{x}\right)^{a} \frac{\exp \left(-\frac{x+y}{2}\right)}{x+y} & 0
\end{array}\right] .
$$

Recall that $a$ and $\sigma$ were defined in (7.1).

It is worth noting that (8.3) involves no special functions. Note also that $\mu=\frac{z-z^{\prime}}{2}$ enters only in the scalar factor $\sigma$.

The formulas (7.2), (7.3), (8.2), (8.3) give a precise description of the process $\widetilde{\mathcal{P}_{z z^{\prime}}}$ and, thus, of the initial process $\mathcal{P}_{z z^{\prime}}$. We consider these formulas as our main result.

\section{Spectral analysis [Part V, $\S 3]$}

In this section we shall diagonalize the operators $K$ and $L$. Denote by $A$ the operator in $L^{2}\left(\mathbb{R}_{+}, d x\right)$ with the kernel

$$
A(x, y)=\frac{\sigma}{\pi}\left(\frac{x}{y}\right)^{a} \frac{\exp \left(-\frac{x+y}{2}\right)}{x+y} .
$$

It is bounded provided that $|a|<\frac{1}{2}$. Let $A^{\prime}$ denote the operator in $L^{2}\left(\mathbb{R}_{+}, d x\right)$ with the transposed kernel

$$
A^{\prime}(x, y)=A(y, x)
$$

Then (8.2) implies that the blocks of the matrix Whittaker kernel are expressed through $A$ and $A^{\prime}$ as follows

$$
\begin{array}{cl}
K_{++}=A A^{\prime}\left(1+A A^{\prime}\right)^{-1}, & K_{--}=A^{\prime} A\left(1+A^{\prime} A\right)^{-1}, \\
K_{+-}=\left(1+A A^{\prime}\right)^{-1} A, & K_{-+}=-\left(1+A^{\prime} A\right)^{-1} A^{\prime} .
\end{array}
$$

Consider the following Sturm-Liouville differential operator on $\left(\mathbb{R}_{+}, d x\right)$ depending on $a$ as a parameter:

$$
\mathcal{D}_{a}=-\frac{d}{d x} x^{2} \frac{d}{d x}+\left(a-\frac{x}{2}\right)^{2} .
$$

We have

$$
\mathcal{D}_{a} A=A \mathcal{D}_{-a}
$$


which implies that $\mathcal{D}_{a}$ (formally) commutes with $A A^{\prime}$ and $K_{++}$while $\mathcal{D}_{-a}$ commutes with $A^{\prime} A$ and $K_{--}$.

Consider the following functions on $\mathbb{R}_{+}$:

$$
f_{a, m}(x)=\frac{1}{x} W_{a, i m}(x), \quad m>0 .
$$

We have

$$
\mathcal{D}_{a} f_{a, m}=\left(a^{2}+\frac{1}{4}+m^{2}\right) f_{a, m}
$$

According to [W], the functions $f_{a, m}$ with $a$ fixed form a continual basis in $L^{2}\left(\mathbb{R}_{+}, d x\right)$ diagonalizing $\mathcal{D}_{a}$. Moreover, the Plancherel formula looks as follows. For good enough functions $f(x)$ and $g(x)$

$$
(f, g)=\frac{1}{\pi^{2}} \int_{0}^{\infty}\left(f, f_{a, m}\right)\left(f_{a, m}, g\right) \cdot \Gamma\left(\frac{1}{2}-a+i m\right) \Gamma\left(\frac{1}{2}-a-i m\right) d m
$$

where $(\cdot, \cdot)$ stands for the inner product in $L^{2}\left(\mathbb{R}_{+}, d x\right)$.

We have

$$
\begin{aligned}
& A f_{-a, m}=\frac{\sigma}{\pi} \Gamma\left(\frac{1}{2}-a+i m\right) \Gamma\left(\frac{1}{2}-a-i m\right) f_{a, m}, \\
& A^{\prime} f_{a, m}=\frac{\sigma}{\pi} \Gamma\left(\frac{1}{2}+a+i m\right) \Gamma\left(\frac{1}{2}+a-i m\right) f_{-a, m} .
\end{aligned}
$$

Returning to the decomposition (8.1), we take $\left\{f_{a, m}\right\}_{m>0}$ as a basis in the first summand $L^{2}\left(\mathbb{R}_{+}, d x\right)$ and $\left\{f_{-a, m}\right\}$ as a basis in the second one.

Then we get a basis in the whole space $L^{2}\left(\mathbb{R}^{*}, d u\right)$ diagonalizing both

$$
L=\left[\begin{array}{cc}
0 & A \\
-A^{\prime} & 0
\end{array}\right] \text { and } K=\left[\begin{array}{cc}
A A^{\prime}\left(1+A A^{\prime}\right)^{-1} & \left(1+A A^{\prime}\right)^{-1} A \\
-\left(1+A^{\prime} A\right)^{-1} A^{\prime} & A^{\prime} A\left(1+A^{\prime} A\right)^{-1}
\end{array}\right]
$$

In particular, we get the diagonalization of $K_{++}$:

$$
K_{++} f_{a, m}=\frac{\cos (2 \pi \mu)-\cos (2 \pi a)}{\cos (2 \pi \mu)+\cos (2 \pi i m)} f_{a, m}
$$

Note that for $a=0$ the integral transform inverse to $f \mapsto\left\{\left(f, f_{a, m}\right)\right\}_{m>0}$ is the Kontorovich-Lebedev transform, see [E2].

The above results show that the operators $K$ and $L$ with $a$ fixed and $\mu$ varying form a commutative family. 


\section{Applications [Part III, Sections 2,5]}

Now we shall give applications of the main results. These applications concern our initial object — the probability measures $P_{z z^{\prime}}$ on $\Omega$.

Theorem V ([Part III, Theorem 5.1 $\left.]^{3}\right)$. Consider $\alpha_{1}, \alpha_{2}, \ldots ; \beta_{1}, \beta_{2}, \ldots$ as random variables on the probability space $\left(\Omega, P_{z z^{\prime}}\right)$. Then with probability 1 there exist the limits

$$
\lim _{k \rightarrow \infty} \alpha_{k}^{1 / k}=\lim _{k \rightarrow \infty} \beta_{k}^{1 / k}=\exp \left(-\frac{\pi \sin (2 \pi \mu)}{2 \mu \sigma^{2}}\right)=\prod_{n \in \mathbb{Z}} e^{-1 /(z-n)\left(z^{\prime}-n\right)}
$$

This result is a kind of the strong law of large numbers. Roughly speaking, it means that both $\alpha_{i}$ 's and $\beta_{i}$ 's decay as geometric progressions with the same exponent. Similar situation occurs for the Poisson-Dirichlet process, see [VS].

The same limit relation as (10.1) holds for $\widetilde{\alpha}_{k}$ 's and $\widetilde{\beta}_{k}$ 's.

The proof of (10.1) is based on the examination of $\widetilde{\rho}_{1}^{\left(z z^{\prime}\right)}$ and $\widetilde{\rho}_{2}^{\left(z z^{\prime}\right)}$, and it incorporates Kingman's argument from [Ki, Section 4.2].

Observe that the exponent of the decay does not change under the shifts $\left(z, z^{\prime}\right) \rightarrow\left(z+N, z^{\prime}+N\right), N \in \mathbb{Z}$. Moreover, the whole process describing the tails of the sequences $\left\{\alpha_{i}\right\}$ and $\left\{\beta_{i}\right\}$ turns out to be invariant with respect to these shifts. This periodicity is quite unexpected: the initial formula (2.3) has no apparent periodicity.

Another application relies on the examination of $\widetilde{\rho}_{1}^{\left(z z^{\prime}\right)}$ alone.

Theorem VI ([Part III, Proposition 2.2]). Let

$$
|\alpha|=\sum_{i=1}^{\infty} \alpha_{i}, \quad|\beta|=\sum_{i=1}^{\infty} \beta_{i}, \quad \psi(\cdot)=\frac{\Gamma^{\prime}(\cdot)}{\Gamma(\cdot)} .
$$

Then

$$
\mathbb{E}|\alpha|=\sin (\pi z) \sin \left(\pi z^{\prime}\right)\left[\frac{z-z^{\prime}}{2 \pi \sin \left(\pi\left(z-z^{\prime}\right)\right)} \frac{z+z^{\prime}-1}{z z^{\prime}}+\frac{\psi\left(-z^{\prime}\right)-\psi(-z)}{\pi \sin \left(\pi\left(z-z^{\prime}\right)\right)}\right],
$$

and $\mathbb{E}|\beta|$ is given by the same formula with $\left(z, z^{\prime}\right)$ replaced by $\left(-z,-z^{\prime}\right)$.

Here $\mathbb{E}$ is the symbol of expectation.

\footnotetext{
${ }^{3}$ The last equality in (10.1) was kindly communicated to us by I. G. Macdonald (letter of December 2, 1998).
} 


\section{Tail process [Part III, Sections 3,4], [Part V, §4]}

Here we study the asymptotic behavior of the process $\widetilde{\mathcal{P}_{z z^{\prime}}}$ near the origin. The starting observation is that

$$
\widetilde{\rho}_{1}^{\left(z z^{\prime}\right)}(u) \sim \frac{c}{|u|}, \quad u \rightarrow 0
$$

where

$$
c=\frac{2 \mu \sigma^{2}}{\pi \sin (2 \pi \mu)}
$$

This asymptotic relation agrees with the rate of decay of $\alpha_{i}$ 's and $\beta_{i}$ 's, see (10.1).

As before, we identify the phase space $\mathbb{R}^{*}$ with the disjoint union of two copies of $\mathbb{R}_{+}$and then in each copy of $\mathbb{R}_{+}$we make the following change of variable

$$
x \mapsto \xi=-c \ln x
$$

Theorem VII ([Part V, Theorem 4.1]). Let $K^{\prime}(\xi, \eta)$ denote the matrix Whittaker kernel on $\mathbb{R}$ obtained from the matrix Whittaker kernel (7.4) by the change of variable (11.3). Then there exists the limit

$$
\lim _{M \rightarrow+\infty} K^{\prime}(\xi+M, \eta+M)=\mathcal{K}(\xi, \eta) .
$$

Here $\mathcal{K}(\xi, \eta)$ is a translation invariant matrix kernel with $\mathcal{K}(\xi, \xi) \equiv 1$,

$$
\mathcal{K}(\xi, \eta)=\left[\begin{array}{ll}
\mathcal{K}_{++}(\xi, \eta) & \mathcal{K}_{+-}(\xi, \eta) \\
\mathcal{K}_{-+}(\xi, \eta) & \mathcal{K}_{--}(\xi, \eta)
\end{array}\right]=\left[\begin{array}{cc}
F(\xi-\eta) & G(\xi-\eta) \\
-G(\eta-\xi) & F(\xi-\eta)
\end{array}\right]
$$

where

$$
\begin{gathered}
F(\zeta)=\frac{B}{A} \frac{\sinh (A \zeta)}{\sinh (B \zeta)} \\
G(\zeta)=\frac{1}{2 \mu \sigma} \frac{(\sin (\pi \mu) \cos (\pi a)) \cosh (A \zeta)+(\sin (\pi a) \cos (\pi \mu)) \sinh (A \zeta)}{\cosh (B \zeta)}
\end{gathered}
$$

and the constants $A, B$ are as follows

$$
B=\frac{\pi \sin (2 \pi \mu)}{4 \mu \sigma^{2}}>0, \quad A=2 \mu B=\frac{\pi \sin (2 \pi \mu)}{2 \sigma^{2}} .
$$

We call the point process on $\mathbb{R} \sqcup \mathbb{R}$ with the correlation functions given by the determinantal formula with the matrix kernel $\mathcal{K}(\xi, \eta)$ the tail process for $\widetilde{\mathcal{P}_{z z^{\prime}}}$. 
By the construction, it describes the behavior of $\widetilde{\alpha}_{i}$ 's and $\widetilde{\beta}_{i}$ 's with large $i$ after the appropriate rescaling.

In particular, the kernel $\mathcal{K}_{++}(\xi, \eta)$ on $\mathbb{R}$ describes the tail of $\left\{\widetilde{\alpha}_{i}\right\}$ alone, and $\mathcal{K}_{--}(\xi, \eta)$ does the same for $\left\{\widetilde{\beta}_{i}\right\}$. Since $\mathcal{K}_{++}=\mathcal{K}_{--}$, the tail properties of $\left\{\widetilde{\alpha}_{i}\right\}$ and $\left\{\widetilde{\beta}_{i}\right\}$ are identical.

The same kernel $\mathcal{K}_{++}(\xi, \eta)$ appears in the scaling limit of the correlation functions for the unlifted process $\mathcal{P}_{z z^{\prime}}$ restricted to $(0,1]$. However, this requires more sophisticated considerations, see [Part II, Sections 4.2, 4.3].

Recall that $\mu$ is either real or pure imaginary. According to this, the constant $A$ is also either real or pure imaginary. In the latter case the hyperbolic sine in the numerator of (11.4) turns into the ordinary sine.

The next result is parallel to Theorem IV.

Theorem VIII ([Part V, Proposition 4.2]). Let $|a|<\frac{1}{2}$. Then

$$
\mathcal{K}=\frac{\mathcal{L}}{1+\mathcal{L}}
$$

where $\mathcal{L}$ is a bounded operator in $L^{2}(\mathbb{R}, d \xi) \oplus L^{2}(\mathbb{R}, d \xi)$ with the kernel

$$
\mathcal{L}(\xi, \eta)=\left[\begin{array}{cc}
0 & \frac{\sigma}{2 \pi} \frac{\exp (-2 a B(\xi-\eta))}{\cosh (B(\xi-\eta))} \\
-\frac{\sigma}{2 \pi} \frac{\exp (-2 a B(\eta-\xi))}{\cosh (B(\eta-\xi))} & 0
\end{array}\right]
$$

where $B$ is as above.

Finally, note that all formulas of this section are invariant under the shifts $\left(z, z^{\prime}\right) \rightarrow\left(z+N, z^{\prime}+N\right), N \in \mathbb{N}$ (or, equivalently, $\left.(a, \mu) \rightarrow(a+N, \mu)\right)$, cf. Section 10 .

\section{Formalism of fermion point processes [Part III, Section 1], [Part V, §1]}

Here we shall discuss elementary general properties of the point processes with determinantal correlation functions [DVJ, Ma1, Ma2].

Let $\mathfrak{X}$ be a phase space with reference measure $d x, K(x, y)$ be a kernel on $\mathfrak{X}$, and $\mathcal{P}$ be a point process on $\mathfrak{X}$ with the correlation functions

$$
\rho_{n}\left(x_{1}, \ldots, x_{n}\right)=\operatorname{det}\left[K\left(x_{i}, x_{j}\right)\right]_{i, j=1}^{n} .
$$

Let $\mathfrak{Y}$ be a subset of $\mathfrak{X}$ such that

$$
\int_{\mathfrak{Y}} \rho_{1}(x) d x<\infty
$$


Then the number of points in $\mathfrak{Y}$ is finite with probability 1 . Moreover, the probability to find exactly $n$ points located in the infinitesimal volumes $d x_{1}, \ldots, d x_{n}$ around points $x_{1}, \ldots, x_{n}$ equals

$$
\pi_{n}\left(x_{1}, \ldots, x_{n}\right) d x_{1} \ldots d x_{n}=\frac{\operatorname{det}\left[L_{\mathfrak{Y}}\left(x_{i}, x_{j}\right)\right]_{i, j=1}^{n}}{\operatorname{det}\left(1+L_{\mathfrak{Y}}\right)} d x_{1} \cdots d x_{n}
$$

Here $L_{\mathfrak{Y}}(x, y)$ is the kernel of the operator $L_{\mathfrak{Y}}$ in $L^{2}(\mathfrak{Y}, d x)$ such that

$$
K_{\mathfrak{Y}}=\frac{L_{\mathfrak{Y}}}{1+L_{\mathfrak{Y}}}
$$

where $K_{\mathfrak{Y}}$ is the operator in $L^{2}(\mathfrak{Y}, d x)$ with the kernel $K(x, y)$ restricted to $\mathfrak{Y}$.

Thus, in case of finite point configurations the operator $L=\frac{K}{1-K}$ has a clear probabilistic meaning - it gives the distribution functions $\pi_{n}$.

It is tempting to apply (12.1) in our case, when the operator $L$ has especially simple form. Unfortunately, we cannot do this for the whole space because the point configurations are infinite. Of course, we can restrict ourselves to an appropriate region $\mathfrak{Y}$, but then the simple form of our $L(x, y)$ will be lost.

Again, in the case of finite configurations, if the space is a disjoint union of two pieces and $L$ is written in block form according to this splitting, then vanishing of the diagonal blocks of $L$ (as in (8.3) and (11.5)) exactly means that the random configuration has equally many points in each of the pieces (see [Part V, Proposition 1.7]). We do not know how to interpret such vanishing in our situation.

\section{Distribution of $\widetilde{\alpha}_{1}$ [Part III, Section 2]}

Here we shall consider the lifted process $\widetilde{\mathcal{P}_{z z^{\prime}}}$ restricted to $\mathbb{R}_{+} \subset \mathbb{R}^{*}$; it is governed by the ' ++ ' block of the matrix Whittaker kernel, see (7.3). We call $K_{++}(x, y)$ the Whittaker kernel.

Note that for any $\tau>0$ the intersection of the random configuration with $\mathfrak{Y}(\tau)=[\tau,+\infty)$ is finite. By $(12.1)$

$$
\operatorname{Prob}\left\{\widetilde{\alpha}_{1}<\tau\right\}=\frac{1}{\operatorname{det}\left(1+L_{\mathfrak{Y}(\tau)}\right)}=\operatorname{det}\left(1-K_{\mathfrak{Y}(\tau)}\right)
$$

Here, following the notation of Section 12, the kernel of $K_{\mathfrak{Y}(\tau)}$ is obtained by restricting $K_{++}(x, y)$ on $[\tau,+\infty)$. As was pointed out by Tracy [T2], a modification of the argument from [TW3, Section V.B] allows to express the Fredholm determinant (13.1) through the Painlevé transcendent V. 


\section{Comparison with random matrices. Degenerations of the Whittaker kernel [Part III, Sections 1,6], [Part V, §5]}

There are a lot of similarities between the processes $\widetilde{\mathcal{P}_{z z^{\prime}}}$ and point processes arising from random matrices. Random matrix theory leads to various kernels: the sine kernel, the Bessel kernel, the Laguerre kernel, etc. (see [Me1, TW1-3]). All of them have the form

$$
\frac{\varphi(x) \psi(y)-\psi(x) \varphi(y)}{x-y}
$$

and so does the Whittaker kernel $K_{++}(x, y)$, see $(7.3)$. Here $\varphi(\cdot)$ and $\psi(\cdot)$ are solutions of certain linear second-order differential equations. The Fredholm determinants of many kernels of this form are expressed through Painlevé transcendents, see [TW3, Sections III and V]. Various kernels (14.1) restricted to suitable intervals commute with Sturm-Liouville operators (see [G], [Me1, §5.3], [TW1], [TW2]). The same is true for the Whittaker kernel restricted to $[\tau,+\infty)$, see [Part III, Section 6].

The Whittaker kernel degenerates to the Laguerre kernel of order $N$ and parameter $\alpha>-1$ if we formally substitute $z^{\prime}=N, z=N+\alpha$, see [Part III, Remark 2.4]. The Bessel kernel is the scaling limit of the Laguerre kernel as $N \rightarrow+\infty$. Similar scaling procedure applied to the Whittaker kernel leads to a two-parametric family of Bessel-type kernels, see [Part V, Theorem 5.1].

Likewise, the stationary kernels from Theorem VII generalize the sine kernel: if $\mu \rightarrow i \infty,(11.4)$ tends to $\frac{\sin (\pi \zeta)}{\pi \zeta}$ irrespective to the behavior of $a$. The kernels $\mathcal{K}_{++}(\xi, \eta)$ have already appeared in $[\mathrm{BCM}, \mathrm{MCIN}]$ in connection with so-called $q$-Hermite ensemble.

As for the matrix Whittaker kernel, it has a similarity with matrix kernels arising from two-matrix ensembles ([EM, Me2, MS]), see [Part IV, Section 3].

\section{Acknowledgements}

We would like to thank Craig A. Tracy for his letter [T2] and for drawing our attention to the works [MTW] and [T1]. We are also grateful to Ian G. Macdonald for valuable remarks (see, in particular, the footnote to Theorem V).

\section{References}

[BCM] C. Blecken, Y. Chen, and K.A. Muttalib, Transitions in spectral statistics, J. Phys. A 27 (1994), L563-L568.

[B] A. Borodin, Biorthogonal ensembles, Preprint, 1998, available via http://xxx.lanl.gov/abs/math/9804027, to appear in Nucl. Phys. B.

[DVJ] D.J. Daley and D. Vere-Jones, An introduction to the theory of point processes, Springer series in statistics, Springer-Verlag,, New York-Berlin, 1988.

[Dy] F.J. Dyson, Statistical theory of the energy levels of complex systems I, II, III, J. Math. Phys. 3 (1962), 140-156, 157-165, 166-175. 
[E1] A. Erdelyi (ed.), Higher transcendental functions, Vols 1-2, McGraw-Hill, New York, 1953.

[E2] A. Erdelyi (ed.), Tables of integral transforms, Vol. 2, McGraw-Hill, New York, 1954.

[EM] B. Eynard and M.L. Mehta, Matrices coupled in a chain. I. Eigenvalue correlations, Preprint, 1997, available via http://xxx.lanl.gov/abs/cond-mat/9710230 .

[G] M. Gaudin, Sur la loi limite de l'espacement des valeurs propres d'une matrice aléatoire, Nucl. Phys. 25 (1961), 447-458.

[KOO] S. Kerov, A. Okounkov, and G. Olshanski, The boundary of Young graph with Jack edge multiplicities, Internat. Math. Res. Notices (1998), no. 4, 173-199; available via http://xxx.lanl.gov/abs/math/9703037.

[KOV] S. Kerov, G. Olshanski, and A. Vershik, Harmonic analysis on the infinite symmetric group. A deformation of the regular representation, C. R. Acad. Sci. Paris Sér. I Math. 316 (1993), 773-778; a detailed version in preparation.

$[\mathrm{KV}] \quad$ S. Kerov and A. Vershik, The Grothendieck group of the infinite symmetric group and symmetric functions with the elements of the $K_{0}$-functor theory of AF-algebras, Representation of Lie groups and related topics (A. M. Vershik and D. P. Zhelobenko, eds.), Adv. Stud. Contemp. Math. 7, Gordon and Breach, New York, 1990, pp. 36114.

[Ki] J.F.C. Kingman, Poisson processes, Oxford University Press, New York, 1993.

[KBI] V.E. Korepin, N.M. Bogoliubov, and A.G. Izergin, Quantum inverse scattering method and correlation functions, Cambridge University Press, Cambridge, 1993.

[L] A. Lenard, Momentum distribution in the ground state of the one-dimensional system of impenetrable bosons, J. Math. Phys. 5 (1964), 930-943.

[Ma1] O. Macchi, The coincidence approach to stochastic point processes, Advances in Appl. Probability 7 (1975), 83-122.

[Ma2] - The fermion process - a model of stochastic point process with repulsive points, Transactions of the Seventh Prague Conference on Information Theory, Statistical Decision Functions, Random Processes and of the Eighth European Meeting of Statisticians (Tech. Univ. Prague, Prague, 1974), Vol. A, Reidel, Dordrecht, 1977, pp. 391-398.

[M] I.G. Macdonald, Symmetric functions and Hall polynomials, 2nd edition, Oxford University Press, New York, 1995.

[MTW] B.M. McCoy, C.A. Tracy, and T.T. Wu, Painlevé functions of the third kind, J. Mathematical Phys. 18 (1977), no. 5, 1058-1092.

[Me1] M.L. Mehta, Random matrices, 2nd edition, Academic Press, Inc., Boston, MA, 1991.

[Me2] ㄴ A method of integration over matrix variables, Comm. Math. Phys. 79 (1981), 327-340.

[MS] M.L. Mehta and P. Shukla, Two coupled matrices: eigenvalue correlations and spacing functions, J. Phys. A. 27 (1994), 7793-7803.

[MCIN] K.A. Muttalib, Y. Chen, M.E.H. Ismail, and V.N. Nicopoulos, New family of unitary random matrices, Phys. Rev. Lett. 71 (1993), 471-475.

[Part I] G. Olshanski, Point processes and the infinite symmetric group. Part I: The general formalism and the density function, Preprint, 1998, available via http://xxx.lanl.gov/abs/math/9804086.

[Part II] A. Borodin, Point processes and the infinite symmetric group. Part II: Higher correlation functions, Preprint, 1998, available via http://xxx.lanl.gov/abs/math 19804087.

[Part III] A. Borodin and G. Olshanski, Point processes and the infinite symmetric group. Part III: Fermion point processes, Preprint, 1998, available via http://xxx.lanl.gov /abs/math/9804088. 
[Part IV] A. Borodin, Point processes and the infinite symmetric group. Part IV: Matrix Whittaker kernel, Preprint, 1998, available via http://xxx.lanl.gov/abs/math /9810013

[Part V] G. Olshanski, Point processes and the infinite symmetric group. Part V: Analysis of the matrix Whittaker kernel, Preprint, 1998, available via http://xxx.lanl.gov/abs /math/9810014 .

[T1] C.A. Tracy, Asymptotics of a $\tau$-function arising in the two-dimensional Ising model, Comm. Math. Phys. 142 (1991), 297-311.

[T2] C.A. Tracy, Whittaker kernel and the fifth Painlevé transcendent, Letter to the authors, April 29, 1998.

[TW1] C.A. Tracy and H. Widom, Level spacing distributions and the Airy kernel, Comm. Math. Phys. 159 (1994), 151-174.

[TW2] L Level spacing distributions and the Bessel kernel, Comm. Math. Phys. 161 (1994), 289-309.

[TW3] , Fredholm determinants, differential equations and matrix models, Comm. Math. Phys. 163 (1994), 33-72.

[VK] A.M. Vershik and S.V. Kerov, Asymptotic theory of characters of the symmetric group, Funct. Anal. Appl. 15 (1981), 246-255.

[VS] A.M. Vershik and A.A. Shmidt, Limit measures arising in the asymptotic theory of symmetric groups I, II, Teor. Verojatnost. i Primenen. 22 (1977), 72-88; 23 (1978), 1, 42-54 (Russian); English translation in Theory of Prob. and its Appl. 22 (1977); 23 (1978).

[W] J. Wimp, A class of integral transforms, Proc. Edinbourgh Math. Soc. (2) 14 (1964), $33-40$.

Department of Mathematics, The University of Pennsylvania, Philadelphia, PA 19104-6395, U.S.A.

E-mail address: borodine@math.upenn.edu

Dobrushin Mathematics Laboratory, Institute for Problems of Information Transmission, Bolshoy Karetny 19, 101447 Moscow GSP-4, RUSSIA

E-mail address: olsh@iitp.ru, olsh@glasnet.ru 